\title{
Minimal Invasive Post Endodontic Monoblock Restorations: I- Richmond Crown: Review
}

\section{Abdulsamee $\mathrm{N}^{*}$ \\ Professor and Head of Dental Biomaterials, Deraya University, Egypt}

*Corresponding author: Nagy Abdulsamee, Professor and Head of Dental Biomaterials, Faculty of Dentistry, Deraya University, Egypt, Email: nagyabdulsamee@gmail.com

\section{Review Article}

Volume 6 Issue 3

Received Date: June 25, 2021

Published Date: July 13, 2021

DOI: $10.23880 /$ oajds- 16000300

\section{Abstract}

In restorative dentistry, restoring poorly fractured endodontically treated teeth is a typical challenge. Such teeth frequently require additional root canal support in the form of a post and core repair. When endodontically treated teeth with lost coronal tooth structure are left untreated for an extended length of time, they may experience supra eruption, drifting, tilting, and rotation of adjacent teeth. Because of the lack of interocclusal space, the dentist may find it difficult to fabricate a crown. To restore normal anatomy, function, and aesthetics, teeth with less remaining crown height should have a post and core followed by a crown. The most challenging patients to treat are those with little inter occlusal clearance and steep incisal guidance. Teeth that have been badly damaged and have little or no crown structure require further retention and support. The Richmond crown is a wonderful option for restoring such teeth. For such circumstances, the Richmond crown is a viable treatment alternative that may be executed with very little incisal clearance to accommodate post, core, and crown thickness. Richmond crown, which comprised a threaded tube in the canal and a screw-retained crown, was first presented in 1878. It was eventually reconfigured as a one-piece dowel and crown when the threaded tube was removed. The current article focuses on Richmond crown restoration and its variations, including diagnosis and treatment planning for such restorations, as well as fabrication techniques.

Keywords: Post; Core; Cast post; Endodontic restorations; Interocclusal; Incisal guidance; Richmond crown; Overjet; CAD/ CAM; Zirconia

Abbreviations: CAM/CAM: Computer-Aided Design and Computer-Aided Manufacturing; PFM: Porcelain Fused to Metal.

\section{Introduction}

Endodontic dentistry has played a vital part in restoring dental function since ancient times, and prosthetic dentistry then restores the function and aesthetics of the tooth $[1,2]$. Many restorative dentists face the challenge of restoring poorly damaged endodontically treated teeth. For the additional retention of the treatment, damaged teeth frequently require additional root canal support [3]. Post and core is a treatment option to increase the retention and resistance form of the tooth in circumstances where the residual crown structure is insufficient to retain a fullcoverage crown [4].

Clinicians have been reporting about the implantation of posts in the roots of teeth to secure restorations for over 250 years. Pierre Fauchard recorded the use of "tenons," which were metal posts screwed into the roots of teeth to hold bridges in place, as early as 1728 [5]. Wood supplanted metal as the post material in the mid-1800s, and the "pivot 
crown," a hardwood post connected to an artificial crown and the root canal, became popular among dentists [5]. The Richmond crown [6], which had a threaded tube placed into the root canal and a screw-retained crown, was launched in 1878.

The "Richmond crown," which is a single-piece postretained crown with a porcelain face, was designed to serve as a bridge retainer. Richmond crown is not a post and core system, but rather a customizable, castable post and crown system $[7,8]$. The post and crown coping are cast as a single unit, with ceramic heated and cemented on the inside canal and over a prepared crown structure with the same insertion path. The ferrule collar is used to strengthen mechanical resistance and retention, as well as to provide anti-rotational properties.

Fracture of the post or root, dislodgement of the postcore assembly, loss of the restorative seal, and periodontal damage are the main concerns with the post and core operation. In patients with a deep bite, which results in maximal oblique forces, the situation may deteriorate further. In such circumstances, substantial core reduction is essential to achieve the appropriate thickness for a metal ceramic crown for improved aesthetics. In such circumstances, the Richmond crown was the restoration of choice [9].

Ideal overjet and overbite are required for restoration of injured anterior teeth with cast metal post and core and allceramic crown [10]. Because of the grey staining generated by the metal substructure and frequent darkening of the free gingival border, using custom cast metal post and core with all-ceramic crowns fails to create a satisfying cosmetic result [11]. A novel approach for restoring such teeth using a modified Richmond's crown made entirely of high-strength zirconia ceramic. The cosmetic and functional rehabilitation of damaged anterior teeth can be aided by an all-ceramic single-unit post-core-crown restoration. Furthermore, the use of computer-aided design and computer-aided manufacturing (CAD/CAM) technologies for fabricating zirconia restorations allows the technique to be completed quickly, precisely, and opening a new avenue for excellent aesthetic restorations [12].

\section{Indications}

Richmond crown is intended for restoring a single tooth that is substantially decaying or severely damaged, with a very low crown height, enhanced deep bite, and decreased overjet [6].

\section{Clinical Cases for Porcelain Fused to Metal Richmond Crown on Anterior Teeth (Conventional Richmond Crown)}

Case 1: A case with broken teeth 11 and 12 was presented. Radiographic assessment of the pulp and periapical regions of teeth (Figure 1A) revealed radiolucency 11, 12 (Figure 1B). On evaluation, it was discovered that the tooth had an enlarged overbite and a decreased overjet, necessitating the placement of a Richmond crown on teeth 11,12 , as shown in (Figure 1), [13].

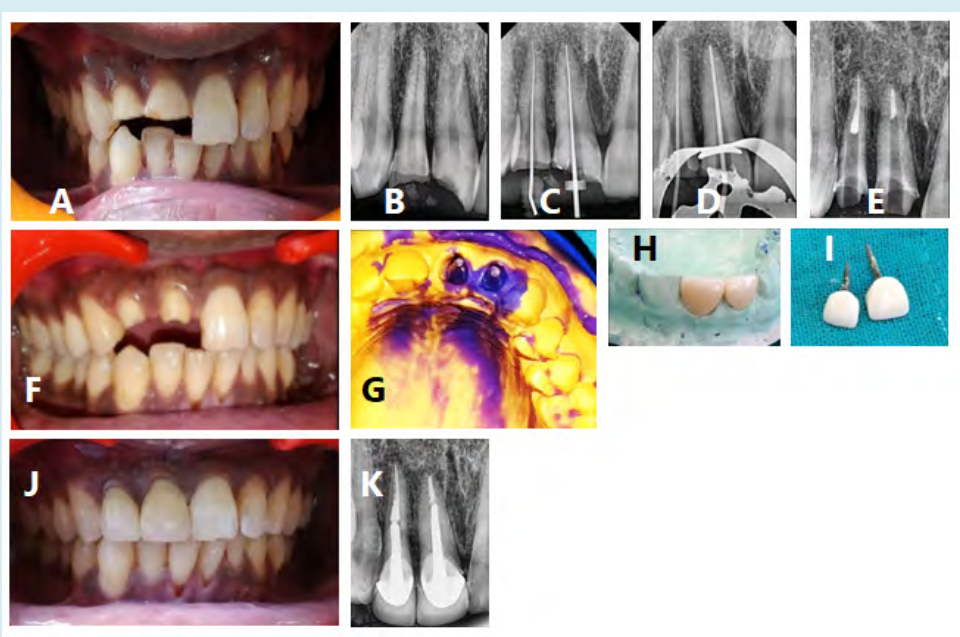

Figure 1 : A) Preoperative Photograph, B) Preoperative radiograph, C) Working length was determined D) The master cone gutta percha radiograph was taken E) After a week of obturation, post space was prepared F) The remaining crown structure was prepared G) Impression of the post hole spaces with an overall impression of the upper arch was made for making stone model, H) Fabricated Richmond crowns on teeth 11 and 12 on the stone model, I) Richmond crowns are ready for cementation J) The Richmond crowns were cemented in situ, and K) Post cementation radiograph of the finished Richmond crowns. 
Case 2: Thirty-five-year-old patient visited a dental clinic in Buguruslan, complaining of the deterioration of previously treated tooth 11 , as well as aesthetic and phonetic issues. The coronal section of tooth 11 was completely missing when the oral cavity was examined (Figure 2A). The coronal portion of the tooth has been completely destroyed, according to the diagnosis. A targeted visiography of tooth 11 with a defect in the coronal region was performed on the patient. The radiograph reveals that the tooth has been treated earlier. The tooth's canal is sealed all the way to the anatomical entrance. At the root canal orifice, an X-ray image revealed a fragment of an anchor metal post (Figure 2B). It was chosen to make a Richmond crown to restore the crown portion of tooth 11 . Removal of the remaining part of the metal anchor pin and unsealing of the root canal by $2 / 3$ of the length of the tooth root (Figure 2C), as well as preparation of the remaining structure of the tooth to intact tissues. We took a silicone impression (Figure 2D). The Richmond crown was created in the dental laboratory (Figure $2 \mathrm{E}-\mathrm{J}$ ), then cemented on the prepared tooth (Figure $2 \mathrm{~K}$ ), and post-pperative X-ray examination was carried out after final cementation to check its quality (Figure 2L) [14].

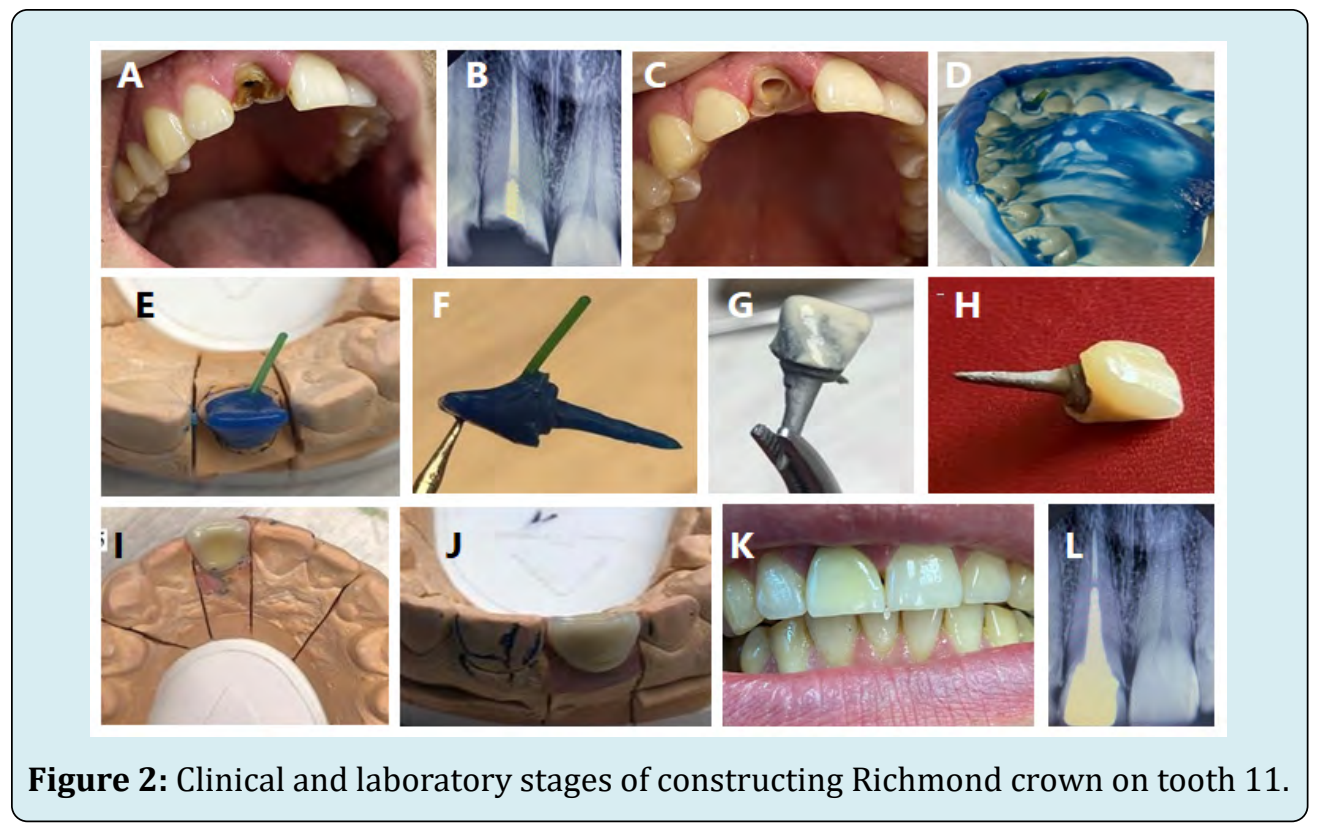

Case 3: A 23-year-old patient complained of the prosthetic crown on tooth 11 dislodging on a regular basis (Figure 3a). Asymptomatic remaining cervical third of 11 had dislodged post and core assembly; nonetheless, centric occlusion was present with deep bite and little overjet, which could be a key cause of prosthesis failure. On radiographic inspection, the root canal was straight, with well-condensed obturation and no periapical alterations around tooth 11.
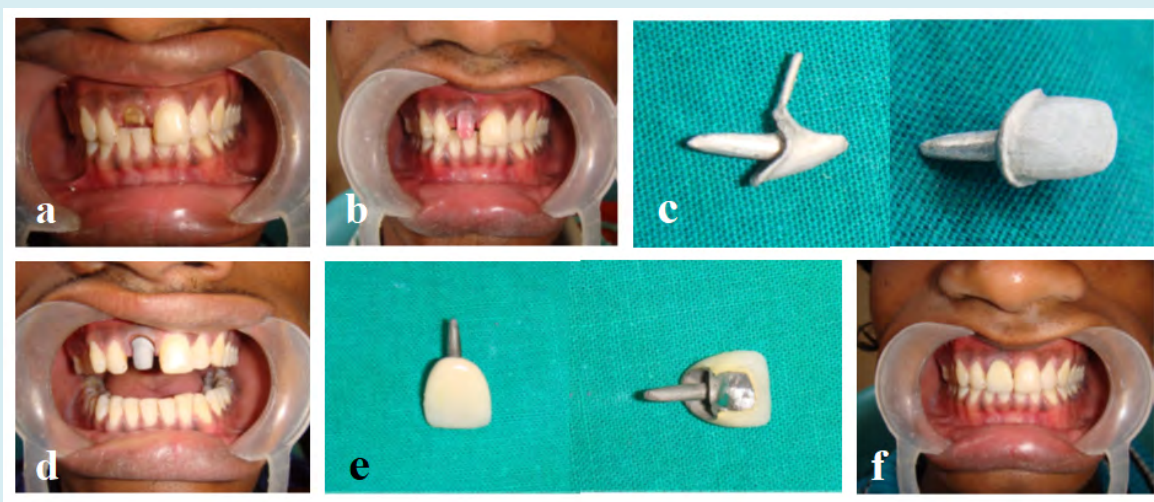

Figure 3: Richmond crown: a) pre-operative view showing dislodged fixed prosthesis on tooth 11 and clinically having very less overjet and deep bite, b) post and core + crown coping build-up in pattern resin, c) casted post and core + crown coping assembly, d) post and core + crown coping assembly checked intra-orally for fit and clearence for ceramic build-up, e) final prosthesis, and f) final prosthesis intra-orally [15]. 
An occlusal model analysis was performed to determine the amount of space available for a post-endodontic restoration to restore the tooth to function, and it was discovered that there was very little overjet to restore the tooth aesthetically, so the Richmond Crown was planned for this case. Following post space preparation, crown structure preparation, post and core fabrication, core structure buildup, and complete coverage extension all over prepared crown like wax pattern for metal coping of metal ceramic crown were performed. The prepared post and core with coping assembly were cast in base metal alloy (Figure 3c), and a metal trial was performed after completing to ensure proper fit (Figure 3d). The finished prosthesis (Figure 3e) was examined for fit and occlusion after the ceramic build up was completed and was cemented (Figure 3f). At a 12-month follow-up, there was no root fracture, no loosening or dislodgement of the post, and no secondary caries [15].

\section{Modified Richmond crowns}

All-ceramic modified Richmond crown fabricated by CAD/CAM: The use of a single-unit all-ceramic post-corecrown zirconia ceramic restoration created using computeraided designing and computer-aided manufacturing (CAD/ CAM) technology offers a unique treatment method for the management of injured anterior teeth with restricted incisal clearance (Figure 4). The use of reinforced zirconia ceramics allows for the creation of long-lasting aesthetic restorations in instances with significant functional loading, and the monobloc effect created by combining the post, core, and crown into a single unit reduces the frequency of failure. Furthermore, using CAD/CAM technology to design and fabricate ceramic restorations allows for faster preparation of these high-strength all-ceramic restorations [16].

A root canal was performed on a broken tooth 21 . (Figure 4a).The typical procedure for post-space preparation was followed. As for an all-ceramic jacket crown with a radial shoulder finish line of $1 \mathrm{~mm}$ width and rounded internal line angles (Figure $4 \mathrm{~b}$ ), shade matching for ceramic restorations was done and tooth preparation was carried out. The post space was imprinted with a two-step putty wash polyvinylsiloxane impression. For accurate opto-electronic scanning, the impression was poured with CAM-Stone $\mathrm{N}$ (Siladent, Goslar, Germany) and digital impressions were made. The CEREC was used to create a three-dimensional reconstruction of the preparation, allowing for a 1-mm decrease of the milled prosthesis on the labial surface and incisal third of the palatal surface for veneering ceramics (Figure 4c). After that, the zirconia coping was milled from a Y-TZP machinable ceramic ingot and sintered at $1500^{\circ} \mathrm{C}$ for 8 hours. A sintered fluorapatite glass ceramic veneer was used to give the milling unit a lifelike appearance on the labial and incisal thirds of the palatal surfaces. Prior to cementation, the completed restoration was assessed for fit, shade, and occlusion, gingival retraction cord was applied, and the restoration was luted with a dual cure composite luting agent. All accessible margins were completed with fine diamonds and polished with rubber tips and diamond polishing paste after the excess luting agent was removed with a knife. Clinically (Figure 4d) and radiographically (Figure 4e), the cemented restoration appeared to provide good cosmetic and functional results [16].

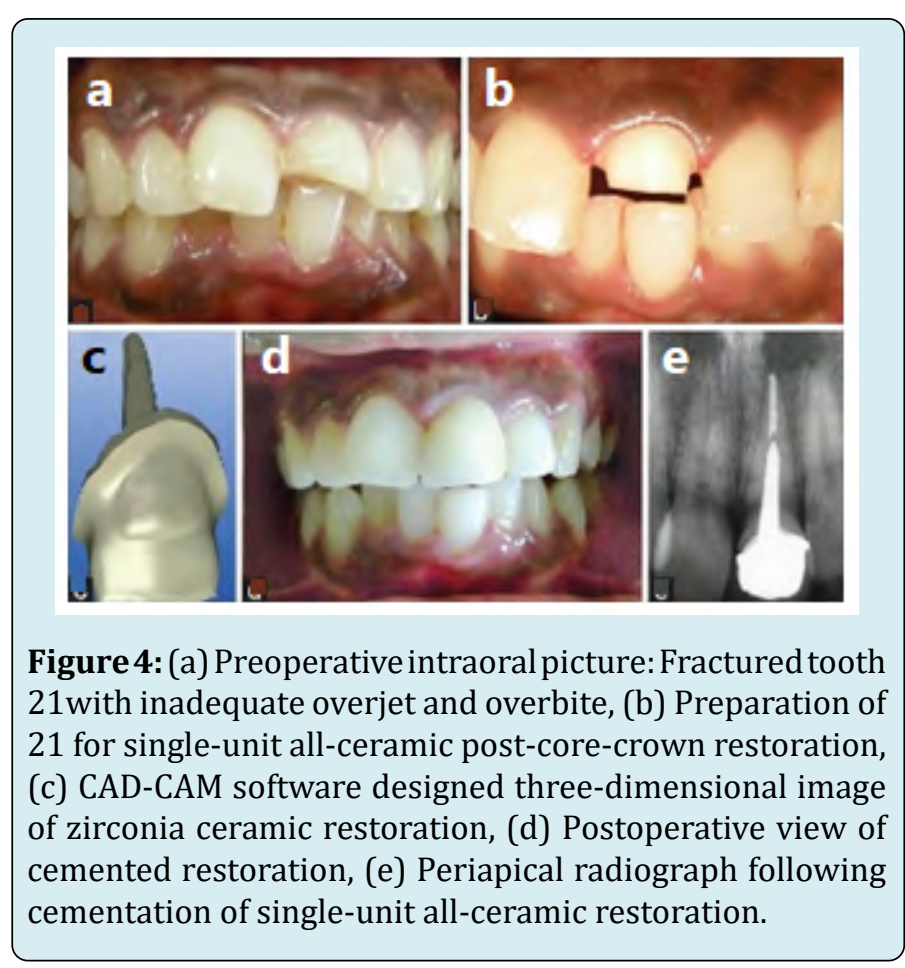

Clinical cases for porcelain fused to metal Richmond crown of posterior teeth: Richmond crown is not a post and core system; rather, it is a customizable, castable post and crown system that is cast as a single item. Cast metal restorations are easy to construct with the help of posts for retention and long-term service. Whenever possible, however, the metal can be masked by using tooth-colored restorations [8].

A 40-year-old patient complained of pain in the lower right back tooth region to the Department of Conservative Dentistry and Endodontics at Vinayaka Mission Sankarachariyar Dental College in Salem. During an examination of the oral cavity, tooth 46 was discovered to have severe caries and a crown fracture at the distolingual cusp (Figure 5A). Deep caries involving the pulp were discovered on an intraoral periapical radiograph (Figure 5B). The root canal treatment was completed, and the obturation was completed with gutta-percha (Figure 5C). Because of the significant loss of tooth structure and lack of occlusal 
clearance for traditional PFM (porcelain fused metal) crowns, the Richmond crown was chosen as a preferable alternative to prefabricated supports [7]. After that, post space was prepared (Figure 5D). The distal canal was filled with light body impression material before a short piece of orthodontic wire, coated with light body, was put in the canal to make the final impression. Following that, a light body was injected around the prepared teeth, putty impression material was poured into the stock tray, and the final imprint was taken (Figure 5E). Impression was then poured with die stone and wax pattern was fabricated. Metal try in was done before ceramic build up then porcelain build up was done and (Figure 5F). Occlusion of Richmond crown was checked on casts (Figure 5G). Finally Richmond crown was cemented (Figure 5H), occlusion was checked intraorally (Figure 5I), and post-operative $\mathrm{x}$-ray for the finished case was taken (Figure 5J).
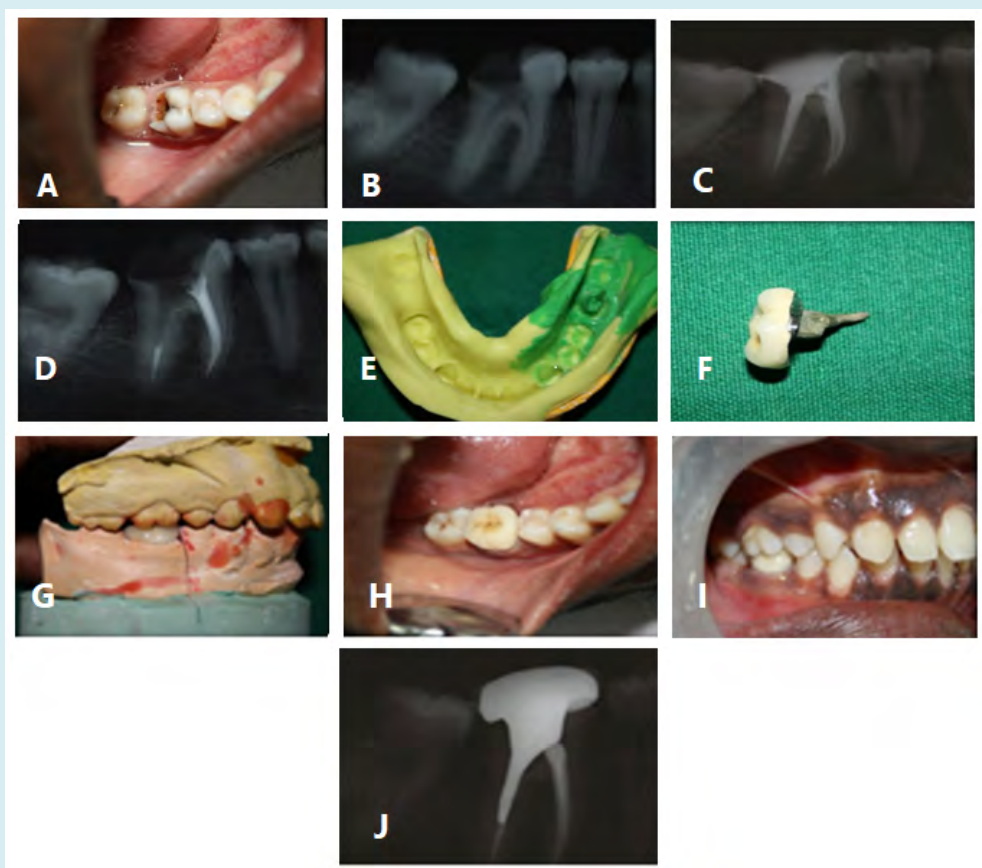

Figure 5: A) Pre-treatment photo, B) Preop-radiograph, C) X-ray after obturation, D) Post hole preparation, E) Impression for post space together with overall impression, F) Rchmond crown, G) Occlusion of Richmond crown on cast, H) Cementation of Richmond crown, I) Post treatment and Richmond crown in occlusion, and J) Post treatment x-ray of Richmond crown insitu.

\section{Advantages of Richmond Crown}

Compared to multiple unit post-core crown restorations, a single unit post-core crown restoration provides a number of advantages. When the post and the core are two different entities, bending of the post under functional pressures stresses the post-core interface, causing the core to separate due to irreversible post deformation [17]. Custom fitting to the root configuration, little or no stress at the cervical margin, high strength, adequate room for ceramic baking, and incisal clearance are all advantages of this design [4]. Caries or crown dislodgement can occur when the core breaks down. Thermal cycling, fatigue loading, and an aqueous environment all work together to test the bond between materials and produce material breakdown over time. For long-term stability, it's preferable to combine the post, core, and crown in one material since it eliminates the cement layer between the core and crown, lowering the risk of cement failure [18]. The single unit restoration helps to generate a "monoblock effect" [16] by reducing the amount of interfaces between components.

\section{Disadvantages of Richmond Crown}

Its disadvantages include being time consuming, requiring multiple appointments, being expensive, having a higher modulus of elasticity than dentin (10 times greater than natural dentin), being less retentive than parallelsided posts, acting as a wedge during occlusal load transfer, and being difficult to retrieve if the ceramic part fractures [19]. Aside from the additional cost of all-ceramic modified Richmond restorations, ceramic posts are difficult to retrieve. As a result, these restorations should only be used in cases when the clinical prognosis is good and the aesthetic value is high [16]. 


\section{Open Access Journal of Dental Sciences}

\section{Discussion}

Endodontic treatment has been used for decades with a good success rate, but the restorative aspect was not well understood. When a significant portion of tooth structure is lost due to fracture, cavities, secondary decay around previous restorations, or during endodontic treatment, the remaining crown structure is insufficient to support a large prosthetic crown [20]. For such instances, the most often utilised method is the post and core procedure [10]. The idea of retaining root (post) retention to increase remaining crown structure (core) and reinforce it is not new. Because the longitudinal axes of the root and the crown diverge, getting them parallel necessitates extensive crown and root pruning. Due to these challenges, a post and core restoration [21] was developed as a separate entity, with an artificial crown cemented over a core and surviving tooth structure.

Recurrent caries, endodontic failure, periodontal disease, post dislodgement, cement failure, post-core separation, crown core separation, loss of post retention, core fracture, loss of crown retention, post distortion, post fracture, tooth fracture, and root fracture have all been identified as major causes of post retained restoration failure. Corrosion of copper supports has also been suggested as a possible cause of root fracture [5].

Richmond crown was introduced in 1878 and was incorporated as a single piece post-retained crown with porcelain face to avoid all of the disadvantages of conventional post and core with separate crown cemented over them. Richmond crown is not a post and core system; rather, it is a customizable, castable post and crown system that is cast as a single item. Casting of the post and crown coping as a single unit, with ceramic baked and cemented on the inside canal and over a prepared crown construction with the same insertion path [8]. In these cases, a Richmond crown is the best option since it requires less crown cutting to bring two axes parallel in a severely decayed tooth and also requires less thickness for the greatest cosmetic effects [10].

Traditional cast metal dowel-cores with porcelain fused to metal (PFM) crowns have been utilised to treat anterior teeth that have been significantly affected by caries or trauma [22]. All-ceramic systems have grown in popularity as the demand for superior aesthetics and patient awareness has increased. Kwiatkowsky and Geller (1989) introduced the cast glass ceramic post and core in 1989 to keep the colour and translucency of pulpless teeth [23]. Due to the brittle nature of glass ceramics, this treatment approach provided great aesthetics but had poor strength and fracture toughness [24].

The strongest and toughest ceramics available for dental application are zirconia ceramic materials, which were launched in the early 1990s. Zirconia is a promising material for endodontic posts because of its exceptional mechanical qualities and aesthetics [25]. Schweiger, et al. (1998) sought to unite the post and core as a single unit by waxing and heat pressing a glass ceramic core directly onto a prefabricated zirconia post [26]. However, because there was still an interface between the zirconia ceramic post and the glass ceramic core, this treatment technique did not achieve a monobloc result.

Compared to multiple-unit post-core-crown restorations, a single-unit post-core-crown restoration made solely of high-strength zirconia ceramic provides a number of advantages. When the post and the core are two distinct entities, bending of the post under functional pressures stresses the post-core interface, causing the core to separate due to irreversible post deformation. Caries or crown dislodgement can occur when the core breaks down. The tooth-post-core-cement-crown complex's bonds are further weakened by the differing coefficients of thermal expansion of the various components. Thermal cycling, fatigue loading, and an aqueous environment all work together to test the bond between materials and produce material breakdown over time. For long-term stability, it is preferable to combine the post, core, and crown in one material [18].

The single unit restoration helps to generate a monobloc effect by reducing the number of interfaces between components. In addition, by minimising the number of clinical procedures needed in fabrication, the CAD/CAM approach has the advantage of minimising chair side time. They are able to minimize inaccuracies and reduce hazards of infectious cross-contamination. Because they can be designed and milled in their soft presintered state and then sintered to provide their physical benefits, machinable zirconia ceramics are ideal for the production of restorations using the CAD/CAM approach [12].

\section{Conclusion}

Although implant popularity is growing by the day, post and core has its importance in replacing severely decaying or damaged teeth since it takes less time and money and produces superior aesthetic outcomes. With a minimally invasive surgery, the Richmond crown improves function and appearance. It is highly recommended in cases when there is insufficient incisal clearance to fit the core, cement, and crown thickness [13]. There are scenarios in which a Richmond crown is advised or contraindicated, as well as characteristics to consider when choosing whether one is the best option for treating a severely decaying or fractured tooth. The Richmond crown can be used to treat a poorly fractured endodontically treated tooth with limited occlusal 
clearance, however it should be utilised with caution [3]. For the cosmetic restoration of severely damaged anterior teeth, an all-ceramic single-unit post-core-crown restoration is a viable therapeutic option. The use of a CAD/CAM process to create an occlusal contact made completely of high-strength zirconia ceramic reduces the risk of the restoration failing due to fracture while also offering an anterior tooth repair with good aesthetics [16].

\section{References}

1. Rosenstiel SF, Land MF and Fujimoto J (2001) Contemporary FixedProsthodontics. $3^{\text {rd }}$ (Edn.), St. Louis Mosby Inc., pp: 380-416.

2. Vârlan C, Dimitriu B, Vârlan V, Bodnar D, Suciu I (2009) Current opinions concerning the restoration of endodontically treated teeth: Basic principles. J Med Life 2(2): 165-172.

3. Chakravarthy Y, Chamarthy S (2015) Richmond crown for restoration of badly mutilated posterior teeth: A case report. J Evid Based Med Healthc 2(30): 4500-4507.

4. Dausage P, Mallikarjuna K, Gupta S, Marya J (2015) Richmond crown esthestics importance for lost tooth structure. Univ J Dent Sci 3: 60-63.

5. Smith CT, Schuman NJ, Wasson W (1998) Biomechanical criteria for evaluating prefabricated post-and-core systems: a guide for the restorative dentist. Quintessence Int 29(5): 305-312.

6. Mishra P, Mantri SS, Deogade S, Gupta P (2015) Richmond crown: A lost state of art. Int J Dent Health Sci 2(2): 448453.

7. Agarwal A, Chadha $M$, Kumar DRV, Somani P, Jain $P$ (2013) Richmond Crown - A Conventional Approach for Restoration of Badly Broken Posterior Teeth. Journal of Dental Peers 1(1): 35-38.

8. Gogna R, Jagadish S, Shashikala K, Keshava Prasad BS (2009) Restoration of badly broken, endodontically treated posterior teeth. J Conserv Dent 12(3): 123-128.

9. Sirimai S, Riis DN, Morgano SM (1999) An in vitro study of the fracture resistance and the incidence of vertical root fracture of pulpless teeth restored with six postand-core systems. J Prosthet Dent 81(3): 262-269.

10. Fernandes AS, Dessai GS (2001) Factors affecting the fracture resistance of post-core reconstructed teeth: A review. Int J Prosthodont 14(4): 355-363.

11. Blatz MB (2002) Long term clinical success of all-ceramic posterior restorations. Quintessence Int 33(6): 415-426.

12. Luthardt RG, Holzhüter MS, Rudolph H, Herold V, Walter $\mathrm{MH}$ (2004) CAD/CAM machining effects on Y-TZP zirconia. Dent Mater 20(7): 655-662.

13. Saraswat A, Nagpal A, Paul R, Pratap R (2019) Esthetic and functional rehabilitation of maxillary anterior teeth by Richmond crown: a case report. HECS Int J Comm Health Med Res 5(3): 68-71.

14. Nestrov AM (2020) Improvement of orthopedic treatment of patients with a complete absence of the crown part of the tooth. Dentistry, Ph.D Thesis of Medical sciences, Samara state Medical University, Ministry of health of Russian.

15. Bhushan K, Sandhu S, Sandhu PK, Bhatia S, Mittal S, et al. (2014) Current status of Richmond crown: an overview on this forgotten treatment. IJRID 4(6): 1-7.

16. Vinothkumar TS, Kandaswamy D, Chanana P (2011) CAD/CAM fabricated single-unit all-ceramic post-corecrown restoration. J Conserv Dent 14(1): 86-89.

17. Libman WJ, Nicholls JI (1995) Load fatigue of teeth restored with cast posts and cores and complete crowns. Int J Prosthodont 8(2): 155-161.

18. Ahn SG, Sorensen JA (2003) Comparison of mechanical properties of various post and core materials. J Korean Acad Prosthodon 41(3): 288-299.

19. Khan F, Mishra SK (2017) Restoration of anterior esthetics with Richmond crown. BLDE Univ J Health Sci 2(2): 109-111.

20. Hudis SI, Goldstein GR (1986) Restoration of endodontically treated teeth: a review of the literature. J Prosthet Dent 55(1): 33-38.

21. Smith CT, Schuman N (1998) Prefabricated post-andcore systems: an overview. Compend Contin Educ Dent 19(10): 1013-1020.

22. Leempoel PJ, Eschen S, De Haan AF, Vant Hof MA (1985) An evaluation of crowns and bridges in general dental practice. J Oral Rehabil 12(6): 515-528.

23. Kwiatkowski S, Geller W (1989) A preliminary consideration of the glass-ceramic dowel post and core. Int J Prosthodont 2(1): 51-55.

24. Rosenblum MA, Schulman A (1997) A review of allceramic restorations. J Am Dent Assoc 128(3): 297-307.

25. Meyenburg KH, Luthy H, Scharer P (1995) Zirconia 
posts: A new all ceramic concept for non-vital abutment teeth. J Esthet Dent 7(2): 73-80.

26. Schweiger M, Frank M, Cramer von Clausbruch S, Holand
W, Rheinberger V (1998) Mechanical properties of a pressed ceramic core to a zirconia post. Quintessence Dent Technol 21: 71-77. 